\title{
Study of determining empirical law of explosion demolition effects of splashed funnel radius in clay medium under water by multivariable regression method
}

\author{
Thang Trong Dam*, Lam Tung Vu \\ Insitute of Techniques for Special Engineering, Military Technical Academy, Vietnam
}

\begin{tabular}{|c|c|}
\hline ARTICLE INFO & ABSTRACT \\
\hline $\begin{array}{l}\text { Article history: } \\
\text { Received } 15^{\text {th }} \text { July } 2020 \\
\text { Revised } 23^{\text {rd }} \text { Sept. } 2020 \\
\text { Accepted } 31^{\text {st }} \text { Oct. } 2020\end{array}$ & $\begin{array}{l}\text { When it comes to blasting in soil, in general, and blasting in clay medium } \\
\text { under water, in particular, typical parameters for destruction effect of } \\
\text { explosion known as splashed funnel based on water depth, the depth of } \\
\text { buried explosives in clay medium and the explosive mass. This relation is }\end{array}$ \\
\hline $\begin{array}{l}\text { Keywords: } \\
\text { Clay medium, } \\
\text { Empirical data, } \\
\text { Explosion under water, } \\
\text { Regression model, } \\
\text { Splashed funnel. }\end{array}$ & $\begin{array}{l}\text { multidimensional and multivariable. Using traditional integation } \\
\text { addressing empirical data still has limits in presenting a general law in } \\
\text { entire domain relating to such relation. Therefore, based on the empirical } \\
\text { results collected from the previuos study, this paper will concentrate to } \\
\text { make a machine learning algorithm building a regression model, finding } \\
\text { the general empirical law about the dependence relation of the radius of } \\
\text { splashed funnel in clay medium under water, based on splashed funnel } \\
\text { and the water depth, the depth of buried explosives in clay medium and } \\
\text { the radius of explosives charges. The efficiency of the model will be } \\
\text { evaluated with correlation coefficent } R^{2} \text { between the calculated values of } \\
\text { funnels and its real values in experiments. Consequently, the model } \\
\text { reached high accuracies which can be applicable in the reality. }\end{array}$ \\
\hline
\end{tabular}

\footnotetext{
${ }^{*}$ Corresponding author

E-mail: thangdt@lqdtu.edu.vn

DOI: 10.46326/JMES.2020.61(5).09
} 


\title{
Tạp chí Khoa học Kỹ thuật Mỏ - Địa chất
}

\section{Nghiên cứu xác định quy luật thực nghiệm về sự biến đổi của bán kính phễu nổ văng trong môi trường đất sét dưới nước theo phương pháp hồi qui đa biến}

\author{
Đàm Trọng Thắng*, Vũ Tùng Lâm \\ Viện Kỹ thuật Công trình Đặc biệt, Học viện Kỹ thuật Quân sự, Việt Nam
}

THÔNG TIN BÀI BÁO T TÓM TẮT

Quá trình:

Nhận bài $15 / 07 / 2020$

Sưa xong 23/9/2020

Chấp nhận đăng 31/10/2020

\section{Tù̀ khóa:}

Hồi quy đa biến,

Môi trường sét,

Nổ dưới nước,

Phễu nổ văng,

Quy luật thực nghiệm.
Khi nổ trong môi trường đất đá nói chung và trong môi trường đất sét dưới nước nói riêng, thông số đặc trung cho tác dụng phá hủy nổ là sự phụ thuộc của bán kính phễu nổ văng vào chiều sâu nước, chiều sâu chồn lượng nổ trong môi trường đất sét và khối lượng thuốc nổ. Mối liên hê này là đa chiều và đa biến. Việc sư dụng công cu giải tích truyền thống khi xủ lý các số liệu thí nghiệm còn hạn chế đưa một qui luật tổng quát trong toàn miền về mối liên hệ trên. Trên cơ sở kết quả thức nghiệm nhận được từ nghiên cúu trước, bài báo này sẽ tập trung phát triển một thuật toán máy hoc để xây dựng một mô hình hồi quy, tìm kiếm quy luật thực nghiệm tổng quát về mối liên hẹ phu thuộc của bán kính phễu nổ văng trong môi trường đất sét dưới nước phu thuộc vào bán kính phễu nổ văng chiều sâu nước, chiều sâu chôn lượng nổ trong môi trường đất sét và bán kính lương nồ. Hiệu quả của mô hình sẽ được đánh giá thông qua hê số tương quan $R^{2}$ giữa giá trị của phễu thu được sau khi tính toán qua mô hình và giá trị thực tế trong thí nghiẹm. Kết quả so sánh cho thấy mô hình có độ chính xác cao, có thể áp dụng vào thực tế.

\section{1. Đặt vấn đề}

Trong thế kỷ XXI thế giới hướng tới khai thác tiềm năng của biển, phục vụ mục đích khai thác khoáng sản và xây dựng các công trình dưới nước. Khi thực hiện các công việc này, thông thường phải sử dụng năng lượng nổ để đào sâu đáy biển, phá đá dưới nước. Tuy nhiên, hiện nay trong lĩnh vực công tác nổ, hệ thống lý thuyết chung và tính toán hộ

\footnotetext{
*Tác giả liên hệ

E - mail: thangdt@lqdtu.edu.vn

DOI: 10.46326/JMES.2020.61(5).09
}

chiếu nổ mới chỉ giải quyết các nhiệm vụ nổ trên cạn. Hệ thống tính toán nổ phá đất đá dưới nước mới chủ yếu theo hướng kế thừa phương pháp nổ trên cạn, chưa có phương pháp tính toán các thông số nổ, thông số phá hủy nổ phụ thuộc vào cả chiều sâu nước (Belin, Dam Trong Thang, 2006; Dam Trong Thang, 2006; Dam Trong Thang và nnk., 2019; Galkin và nnk., 1987; Novikov và nnk., 1984; Gorodinov, 1993; Tavrivov, 1949). Chính vì lý do trên việc nghiên cứu qui luật thực nghiệm về sự phụ thuộc của bán kính phễu nổ vào chiều sâu chôn lượng nổ, chiều sâu nước và bán kính lượng nổ là một hướng đi có tính cấp thiết, có ý nghĩa khoa học và thực tiễn. 
Việc thiết lập các qui luật thực nghiệm về các mối liên hệ giữa các thông số ở mức đa chiều đa biến rất khó giải quyết bằng phương pháp hồi qui truyền thống. Hiện nay, nhũng thành tựu nghiên cứu trong lĩnh vực trí tuệ nhân tạo đang được áp dụng rất thành công vào nhiều ngành khoa học và kỹ thuật. Trong đó, máy học và học sâu là một hướng tiếp cận rất hiệu quả xét riêng về lĩnh vực khoa học dữ liệu. Chính vì vậy, bài báo này tập trung phát triển một thuật toán máy học để xây dựng một mô hình hồi quy nhằm tìm kiếm quy luật phụ thuộc của bán kính phễu phá hủy văng vào chiều sâu chôn lượng nổ trong đất sét, chiều sâu nước và bán kính lượng nổ.

\section{Mô tả nội dung, kết quả nghiên cứu thực nghiệm}

\subsection{Mô tả nội dung thí nghiệm}

Sơ đồ chung của thiết bị mô hình được thể hiện trên Hình 1. Các vụ nổ mô hình được thực hiện trong các bể kim loại đường kính $0,7 \mathrm{~m}$, chiều cao 0,9 m, được đổ đầy nước ngọt đến bề mặt nước đảm bảo độ sâu theo yêu cầu. Các lượng nổ tập trung được sử dụng bằng thuốc nổ Ten, với khối lượng tương đương thuốc nổ TNT (hoặc Amonit số 6JV) 0,5 g, được nhồi trong vỏ nhôm. Bán kính lượng nổ tương ứng $5 \mathrm{~mm}$. Vật liệu sử dụng trong mô hình nổ là đất sét có chiều dày $0,5 \mathrm{~m}$ (Hình 1 ). Trọng lượng thể tích của đất sét ở trạng thái bão hòa nước là $1,768 \mathrm{~kg} / \mathrm{m}^{3}$, tốc độ sóng âm lan truyền trong đất sét là $1768 \mathrm{~m} / \mathrm{s}$. Kích nổ các lượng nổ mô hình bằng phương pháp điện với kíp nổ siêu nhỏ từ azotua chì, được lắp bên trong vỏ lượng nổ.

Chiều sâu tuyệt đối của nước trong các thí nghiệm được dao động $0 \div 120 \mathrm{~mm}$ hoặc đến 350 mm, chiều sâu lớp nước biểu thị tương đối so với bán kính lượng nổ tương ứng là $0 \div 24 \mathrm{~mm}$ hoặc đến $70 \mathrm{~mm}$. Chiều sâu tuyệt đối chôn lượng nổ tập trung trong đất sét được dao động $0 \div 55 \mathrm{~mm}$, chiều sâu đặt thuốc biểu thị tương đối so với bán kính lượng nổ tương ứng là $0 \div 11 \mathrm{~mm}$. Dạng chung của phễu nổ văng và vùng nén khi nổ các lượng nổ tập trung với sự dao động của chiều sâu nước và chiều sâu chôn lượng nổ trong đất sét từ trị số nhỏ đến trị số lớn được mô tả trong Hình 2.

\subsection{Lựa chọn các thông số đồng dạng để thiết lập qui luật thực nghiệm}

Để thiết lập sự phụ thuộc của bán kính phếu nổ $R_{B}$ dựa trên các tham số chiều sâu chôn lượng nổ trong đất sét $W$, chiều sâu mực nước $\mathrm{h}$ và bán kính lượng nổ $\mathrm{r}$, sử dụng nguyên tắc đồng dạng để đưa vào các thông số đồng dạng vô thứ nguyên sau (Dam Trong Thang, 2006; Baum và nnk., 1975; Sedop, 1954):

- Chiều sâu đặt thuốc tương đối của lượng nổ tập trung là tỉ số giữa chiều sâu chộn thuốc trong môi trường đất sét $W$ và bán kính lượng nổ $r$ : $\bar{W}=$ $W / r$;

- Chiều sâu mực nước tương đối là tỉ số giữa chiều sâu mực nước và bán kính lượng nổ $r: \bar{h}=$ $h / r$;

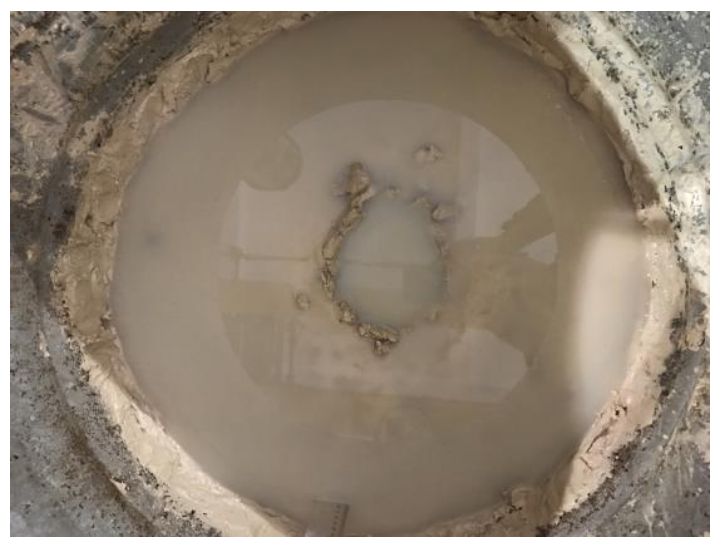

(b)

Hình 1. Sơ đồ chung của thiết bị trong mô hình hóa các vụ nổ, các lượg nổ tập trung trong đất sét dưới nước (a); ảnh chụp kết quả sau nổ (b). (1- bề mặt nền đất; 2- bể kim loại; 3- đất sét; 4- bề mặt nước; 5- mức tương đương của bề mặt tự do khi thay thế nước về đất sét; 6- lượng nổ). 
- Bán kính miệng phễu văng tương đổi là tỉ số giữa bán kính phễu nổ văng $R_{B}$ và bán kính lượng nổ $r: \overline{R_{B}}=R_{B} / r$.

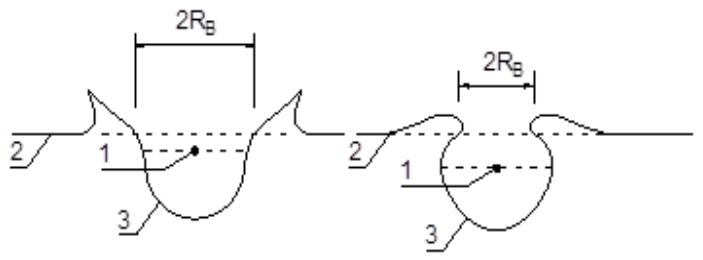

Hình 2. Dạng chung của phễu nổ văng và vùng nén khi nổ trong đất sét ngập nước bằng các lượng nổ tập trung. (1- lượng nổ chôn trong đất sét; 2- bề mặt đất tự nhiên; 3-phễu hoặc vùng nén).

\subsection{Kết quả nghiên cứu thực nghiệm}

Các số liệu thực nghiệm nhận được đã được các tác giả Đàm Trọng Thắng và nnk. (2019) phân tích và thiết lập các quy luật thực nghiệm như sau:

* Khi chiều sâu đặt thuốc trong đất sét $W / r=1$ :

$$
\begin{gathered}
\frac{R_{B}}{r}=-0,0187\left(\frac{h}{r}\right)^{2}+0,1641\left(\frac{h}{r}\right)+14,51 \\
R^{2}=0,9542 \text {, tại } 0 \leq h / r \leq 24 \\
\frac{R_{B}}{r}=\frac{R_{B h}}{r} \approx 7,2, \Delta= \pm 3 \% ; \\
\text { tại } 24 \leq h / r \leq 60
\end{gathered}
$$

* Khi chiều sâu đặt thuốc trong đất sét $W / r=3$ :

$$
\begin{gathered}
\frac{R_{B}}{r}=14,086 . e^{-0,029 \frac{h}{r}} \\
R^{2}=0,9821 ; \operatorname{taii} 0 \leq h / r \leq 24 \\
\frac{R_{B}}{r}=\frac{R_{B h}}{r} \approx 7 \\
\Delta= \pm 14 \% ; \text { taii } 24 \leq h / r \leq 60
\end{gathered}
$$

* Khi chiều sâu đặt thuốc trong đất sét $W / r=7$ :

$$
\begin{gathered}
\quad \frac{R_{B}}{r}=12,11 \cdot e^{-0,026 \frac{h}{r}} ; \\
R^{2}=0,9398 ; \operatorname{tai} 0 \leq h / r \leq 24 ; \\
\frac{R_{B}}{r}=\frac{R_{B h}}{r} \approx 6,5 ; \\
\Delta= \pm 3 \% ; \text { taii } 24 \leq h / r \leq 60 ;
\end{gathered}
$$

* Khi chiều sâu đặt thuốc trong đất sét $W / r=11$ :

$$
\begin{gathered}
\frac{R_{B}}{r}=10,924 \cdot e^{-0,092 \frac{h}{r} ;} \\
R^{2}=0,8869 ; \text { taii } 0 \leq h / r \leq 24 ;
\end{gathered}
$$

Trong đó: $R_{B}$ - bán kính phễu nổ văng, $R_{B h}$ - Bán kính phễu nổ văng tương ứng đạt ở trị số bão hòa, $h$ - chiều sâu nước, $r$ - bán kín lượng nổ, $R^{2}$ : hệ số tương quan, $\Delta$ - Độ chênh lệch giá trị của dữ liệu thực nghiệm bán kính phễu nổ văng xung quanh giá trị bão hòa.

Phân tích hệ thống lý thuyết tác dụng cơ học nổ trong môi trường đất đá cho phép đưa ra nhận xét sau (Gankin và nnk., 1987; Baum và nnk., 1975; Pokropski và nnk., 1957; Cedov, 1954; Kriukov, 1985; Gorodinov, 1993; Kutuzov và nnk., 1970; Josef Henrych, 1979): công thức thực nghiệm (7) rút ra từ mức chiều sâu đặt thuốc trong đất sét $W / r$ $=11$ đã dần đạt đến trạng thái bão hòa, tức miền nổ ngầm. Do đó, có thể tiến hành định hướng mô hình bằng cách bổ sung thêm các mẫu cho các mức đặt thuốc $W / r=15,19, \ldots$ mà vẫn sử dụng các công thức bão hòa từ mức đặt thuốc $W / r=11$. Xét ở tính qui luật tác dụng phá hủy nổ, thì việc bổ sung bộ số liệu này hoàn toàn đúng đắn.

Có nghĩa là khi chiều sâu đặt thuốc trong đất sét $W / r>11$ thì ta có:

$$
\frac{R_{B}}{r}=\frac{R_{B h}}{r}=0 \text { tại } h / r>24 ;
$$

Từ công thức (1) $\div(7)$, có thể nhận thấy rằng khi $W / r$ tăng thì giới hạn bão hòa sẽ đúng tại ngưỡng $\mathrm{h} / \mathrm{r}$ giảm dần về 0 . Tức là đến một ngưỡng $W / r$ nào đó thì với mức nước bằng không, quá trình nổ lượng nổ sẽ chuyển thành nổ ngầm và việc hình thành phễu sẽ không xảy ra. Do thí nghiệm chưa xác định ngưỡng $W / r$ này nên công thức (8) sẽ mang tính chất định hướng mô hình (đồng nghĩa với việc định hướng thực hiện thí nghiệm).

Sử dụng các công thức thực nghiệm trên cho các khoảng $h / r$ từ 0 đến $60, W / r=1 ; 3 ; 7 ; 11 ; 15 ; 19$, kết quả được thể hiện bằng hình vẽ biểu diễn trường dữ liệu trong không gian 3 chiều và được trình bày trong phần 3 của bài báo.

\section{Phân tích và thiết lập mô hình hồi quy đa biến}

\subsection{Co' sở toán học}

Hồi quy là một phương pháp thống kê phân tích mối quan hệ giữa biến phụ thuộc y với một hay nhiều đặc trưng $x_{j}$. Như vậy, bài báo sẽ xây dựng một hàm giả thiết $h_{\theta(x)}$ là một hàm theo $x_{j}$ với các hệ số là các tham số ước lượng $\theta_{j}$. Hàm mất mát được sử dụng là sai số bình quân phương (MSE), các tham số ước lượng $\theta_{j}$ được cập nhật sau mỗi vòng lặp (epoch) sử dụng toàn bộ bộ dữ liệu huấn luyện thông qua kỹ thuật "xuống dốc" (gradient 
descent). Vấn đề "quá mức" (overfitiing) của mô hình được xử lý bằng hồi quy ridge thông qua siêu tham số $\lambda$. Mỗi đặc trưng của dữ liệu trước khi được đưa vào huấn luyện phải trải qua quá trình chính quy hóa (standardisation) thông qua kỳ vọng $\mu_{j}$ và độ lệch chuẩn $\sigma_{j}$ của mỗi đặc trưng (Vu Huu Tiep, 2018; Andrew, 2011).

Bộ số liệu được chia thành 3 phần, phần 1 là bộ huấn luyện dùng để tối ưu các tham số $\theta_{j}$, phần 2 là bộ xác thực chéo để tìm mô hình với các siêu tham số (hyper parameters) điều chỉnh mức độ phù hợp của hàm giả thiết, phần 3 là bộ kiểm tra để đánh giá hiệu suất của mô hình tìm được. Mức độ hiệu quả của mô hình sẽ được đánh giá qua hệ số tương quan $R^{2}$.

\subsection{Lập mô hình dự đoán bán kính phễu nổ văng tương đối $\overline{R_{B}}$}

Mô hình được xây dựng với ngôn ngữ lập trình Python 3.7 theo bản phân phối miễn phí và nguồn mở Anaconda 3.

Các kích thước phễu nổ chỉ phụ thuộc vào $\bar{W}$ và $\bar{h}$ tương ứng với chỉ 2 chiều dữ liệu nên có thể hiển thị trường dữ liệu trong không gian 3 chiều với các trục $x, y, z$ tương ứng với $\bar{W}, \bar{h}$ và $\overline{R_{B}}$ (chiều sâu đặt thuốc qui đổi, chiều sâu nước qui đổi và bán kính miệng phễu qui đổi) như sau:

Giả sử sự phụ thuộc của $\overline{R_{B}}$ vào các thông số thí nghiệm là không quá phức tạp, đồng nghĩa với các giá trị của các biến $\bar{W}$ và $\bar{h}$ là độc lập với nhau.

Một hàm giả thiết dự đoán sự phụ thuộc của $\overline{R_{B}}$ tương ứng với bậc $\mathrm{n}$ của các biến có dạng như sau:

$$
\begin{gathered}
\overline{R_{B}}=\theta_{0}+\theta_{1} \cdot \frac{h}{r}+\theta_{2} \cdot \frac{W}{r}+\theta_{3} \cdot\left(\frac{h}{r}\right)^{2}+\theta_{4} \cdot\left(\frac{W}{r}\right)^{2}+ \\
\ldots+\theta_{2 d-1} \cdot\left(\frac{h}{r}\right)^{d}+\theta_{2 d} \cdot\left(\frac{W}{r}\right)^{d}
\end{gathered}
$$

Trong đó: $d$ - số bậc tương ứng với độ phức tạp của mô hình; $\overline{R_{B}}$ - bán kính miệng phễu nổ qui đổi; $\theta_{j}$ - các tham số ước lượng.

Dưới dạng ma trận, có thể viết lại như sau:

Trong đó:

$$
\left\{\overline{R_{B}}\right\}=[X] .\{\theta\}
$$

$$
[X]=\left[\{1\}\left\{\frac{h}{r}\right\}\left\{\frac{W}{r}\right\} \cdots\left\{\frac{h}{r}\right\}^{d}\left\{\frac{W}{r}\right\}^{d}\right]
$$

$[X]$ - ma trận $m \times n$ gồm các thành phần là các véc tơ cột; $m$ là số mẫu huấn luyện; $n=2 d+1$ - số đặc trưng của mô hình; $d$ - số bậc của mô hình; $\{\theta\}$ là véc tơ cột $n \times 1 ;\left\{\overline{R_{B}}\right\}$ - véc tơ cột $m \times 1$.

Cụ thể như công thức (12).

Trong đó: (1), (2),..., (i), ..., $(m)$ là thứ tự của mẫu trong bộ số $(i=1 \div m)$; số bậc mô hình $j=1 \div d$.

Với bộ số liệu từ các công thức thực nghiệm có tổng cộng 316 mẫu, bộ huấn luyện được chọn là 190 mẫu, bộ xác thực chéo và bộ kiểm tra là 63 mẫu/bộ. Để đảm bảo mô hình xây dựng được khách quan thì tỷ lệ chia mẫu cho từng bộ số liệu trên sẽ được chọn ngẫu nhiên.

Chọn số bậc của mô hình $d=4$, tiến hành tính toán sai số trên cả 2 bộ huấn luyện và xác thức chéo với $\lambda$ tăng dần, tham số $\lambda$ được lựa chọn sao cho sai số của cả hai bộ huấn luyện và xác thực chéo đều nhỏ nhất, với $d=4, \lambda=0.01$ là phù hợp. Kết quả được biểu thị ở Hình 4 .

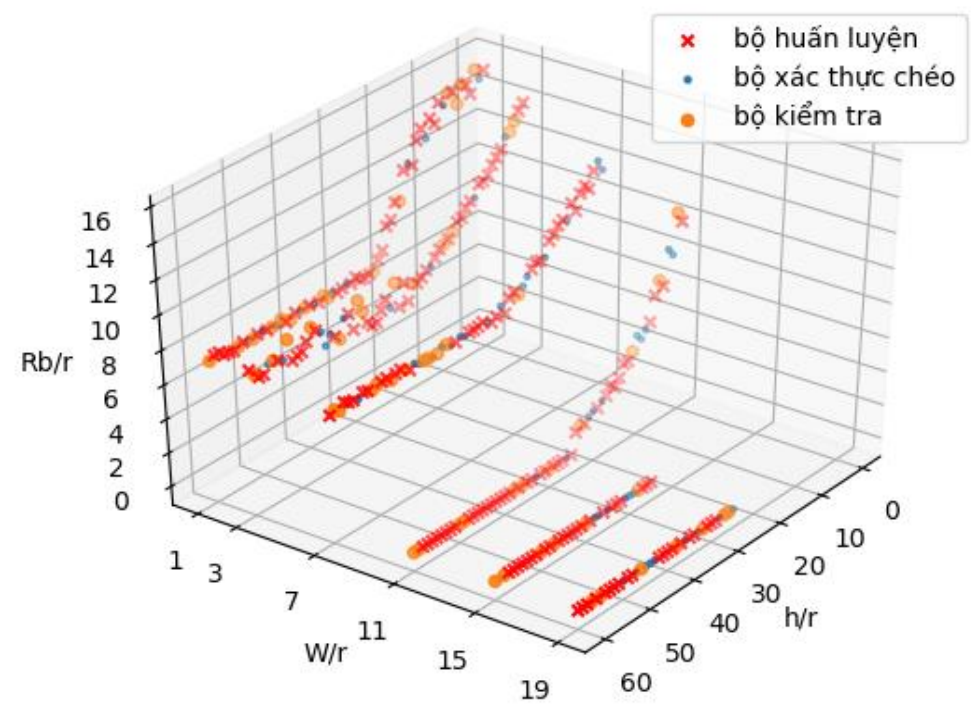

Hình 3. Dũ liệu đã xử lý tù̀ công thức thực nghiệm $(1 \div 8)$ giữa $\overline{R_{B}}$ và $\bar{W}, \bar{h}$. 
$\left\{\begin{array}{c}\bar{R}_{B}^{(1)} \\ \bar{R}_{B}^{(2)} \\ \vdots \\ \bar{R}_{B}^{(i)} \\ \vdots \\ \bar{R}_{B}^{(m)}\end{array}\right\}_{m \times 1}=\left[\begin{array}{ccccccccc}1 & \left(\frac{h}{r}\right)_{(1)} & \left(\frac{W}{r}\right)_{(1)} & \cdots & \left(\frac{h}{r}\right)_{(1)}^{j} & \left(\frac{W}{r}\right)_{(1)}^{j} & \cdots & \left(\frac{h}{r}\right)_{(1)}^{d} & \left(\frac{W}{r}\right)_{(1)}^{d} \\ 1 & \left(\frac{h}{r}\right)_{(2)} & \left(\frac{W}{r}\right)_{(2)} & \cdots & \left(\frac{h}{r}\right)_{(2)}^{j} & \left(\frac{W}{r}\right)_{(2)}^{j} & \cdots & \left(\frac{h}{r}\right)_{(2)}^{d} & \left(\frac{W}{r}\right)_{(2)}^{d} \\ \vdots & \vdots & \vdots & \ddots & \vdots & \vdots & \ddots & \vdots & \vdots \\ 1 & \left(\frac{h}{r}\right)_{(i)} & \left(\frac{W}{r}\right)_{(i)} & \cdots & \left(\frac{h}{r}\right)_{(i)}^{j} & \left(\frac{W}{r}\right)_{(i)}^{j} & \cdots & \left(\frac{h}{r}\right)_{(i)}^{d} & \left(\frac{W}{r}\right)_{(i)}^{d} \\ \vdots & \vdots & \vdots & \ddots & \vdots & \vdots & \ddots & \vdots & \vdots \\ 1 & \left(\frac{h}{r}\right)_{(m)} & \left(\frac{W}{r}\right)_{(m)} & \cdots & \left(\frac{h}{r}\right)_{(m)}^{j} & \left(\frac{W}{r}\right)_{(m)}^{j} & \cdots & \left(\frac{h}{r}\right)_{(m)}^{d} & \left(\frac{W}{r}\right)_{(m)}^{d}\end{array}\right]_{m \times(2 d+1)} \times\left\{\begin{array}{c}\theta_{0} \\ \theta_{1} \\ \vdots \\ \theta_{2 j} \\ \vdots \\ \theta_{2 d}\end{array}\right\}_{(2 d+1) \times 1}$
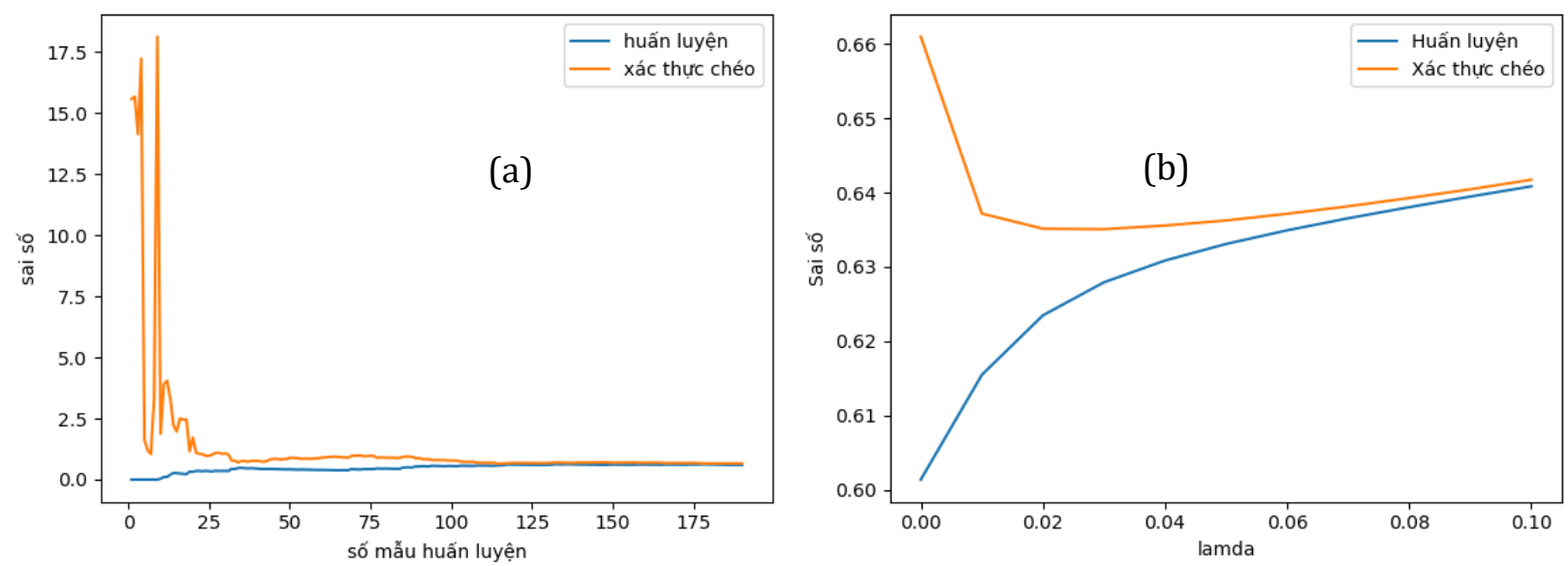

Hình 4. Tương quan sai số giữa bộ huấn luyện và bộ xác thực chéo bậc 4 theo số mẫu huấn luyện (a) và theo $\lambda$ (b) khi lập mô hình $\overline{R_{B}}$.

Vì mô hình được học trực tiếp từ bộ huấn luyện nên sai số ở bộ này luôn thấp, kết quả là đường màu xanh trên Hình 4a. Một bộ các tham số ước lượng được tính và hiệu chỉnh dần với từng lần tăng số mẫu huấn luyện, với mỗi lần hiệu chỉnh sẽ được sử dụng vào để tính sai số với bộ xác thực chéo, kết quả là đường màu cam trên Hình 4 . Độ chụm của 2 đường càng lớn thể hiện mức độ khái quát của mô hình càng lớn; mức tiệm cận của hai đường càng về 0 thể hiện mức độ phù hợp của mô hình càng cao.

Với kết quả là độ chụm và mức tiệm cận 0 đều lớn, chứng minh mô hình tốt. Tiếp tục sử dụng mô hình này để tiến hành dự đoán toàn bộ các giá trị của $\overline{R_{B}}$ rồi xây dựng một trường bề mặt biểu thị quy luật của $\overline{R_{B}}$ với các biến trong không gian 3 chiều. Đánh giá hiệu quả của mô hình dựa trên hệ số tương quan $R^{2}$ có:

$$
R^{2}=1-\frac{\sum_{i=1}^{m}\left(\frac{R_{B}^{(i)}}{r}-\frac{\widehat{R_{B}^{(l)}}}{r}\right)^{2}}{\sum_{i=1}^{m}\left(\frac{R_{B}^{(i)}}{r}-\frac{\overline{R_{B}}}{r}\right)^{2}}
$$

Trong đó: $\frac{R_{B}^{(i)}}{r}$ - giá trị bán kính miệng phễu nổ quy đổi thực tế ở lần nổ thứ i trong bộ kiểm tra; $\frac{\overline{R_{B}}}{r}$ - giá trị bán kính miệng phễu nổ quy đổi trung bình trên thực tế của bộ kiểm tra; $\frac{\widehat{R_{B}^{(l)}}}{r}$ - giá trị bán kính miệng phễu nổ quy đổi dự đoán được từ mô hình ở lần nổ thứ $\mathrm{i} ; \mathrm{m}$ là tổng số mẫu thực tế.

Kết quả có $R^{2}=0.934$.

Tuy nhiên trực quan có thể thấy rằng, trong khoảng $\bar{W}>11$ và $0 \leq \bar{h} \leq 24$, quy luật không phù hợp. Nguyên nhân là do chưa có dữ liệu tại vùng bão hòa tuyệt đối ứng với từ một giá trị $\bar{W}$ trở đi, nổ om xảy ra ngay tại $\bar{h}=0$. Trong nghiên cứu trước của các tác giả (Dam Trong Thang và nnk., năm 2019), mức tuyệt đối này được chỉ ra ở tại $\bar{W}=28$, dữ liệu mới như sau (Hình 6).

Kết quả trên đồ thị Hình 6 cho thấy $\lambda=0$ là phù hợp, bề mặt biểu thị quy luật $\overline{R_{B}}$ như Hình 7 .

Bộ số liệu mới có tổng cộng 377 mẫu, bộ huấn luyện được chọn là 227 mẫu, bộ xác thực chéo và bộ kiểm tra là 75 mẫu/bộ. Tiếp tục sử dụng mô hình có $d=4$, biến độc lập cho bộ số định hướng mới được (Hình 7). 


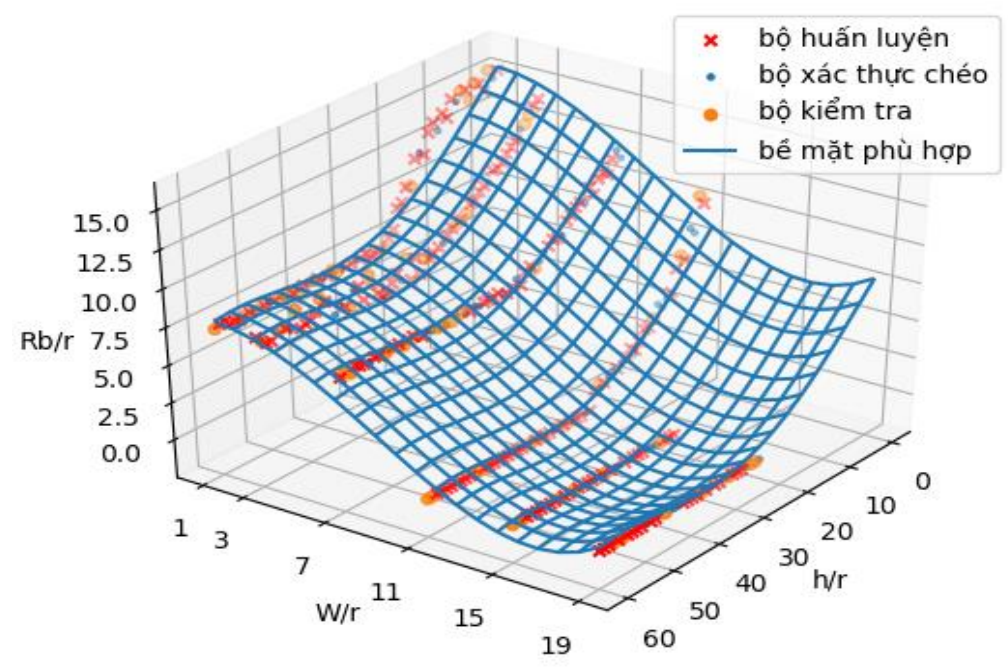

Hình 5. Biểu diễn mô hình $\overline{R_{B}}$ bằng một bề mặt phù hợp.

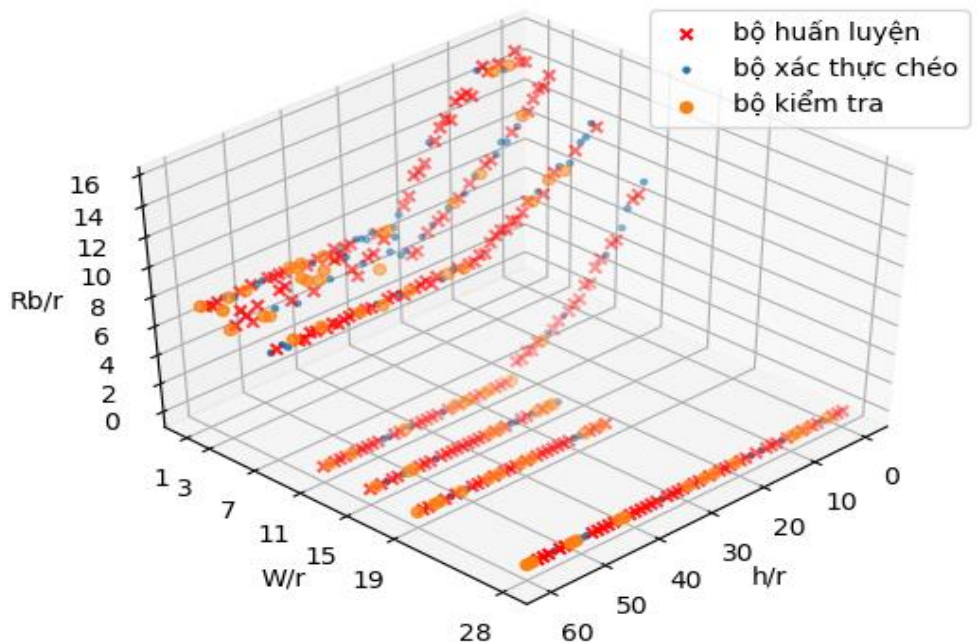

Hình 6. Dũ liệu đã xử lý tù̀ công thức thực nghiệm (1)-(8) giữa $\overline{R_{B}}$ và $\bar{W}, \bar{h}$ sau khi được định hướng bằng dũ liệu mới.
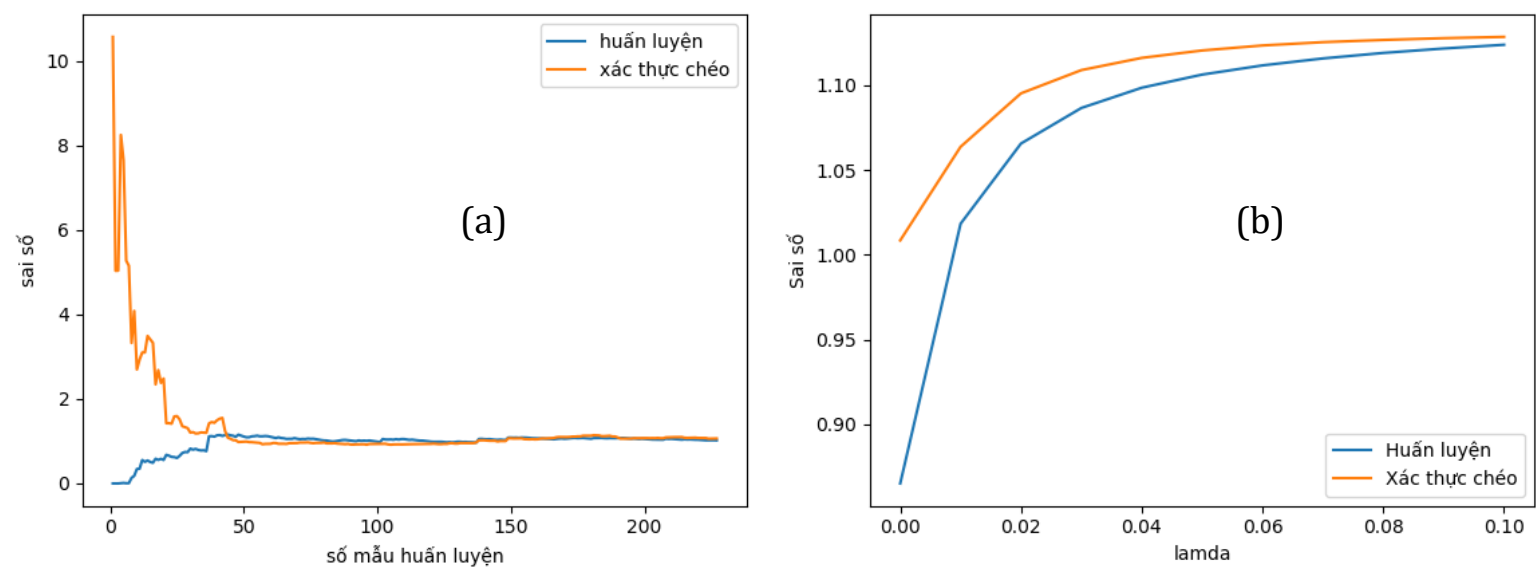

Hình 7. Tương quan sai số giũa bộ huấn luyện và bộ xác thực chéo bậc 4 theo số mẫu huấn luyện (a) và theo $\lambda$ (b) theo dũ liệu định hướng khi lập mô hình $\overline{R_{B}}$. 
Từ (13), kết quả có $R^{2}=0.902$. Với dữ liệu mới, hệ số tương quan $R^{2}$ giảm, cho thấy quy luật đã trở nên phức tạp hơn nên việc coi các biến là độc lập không còn phù hợp, mặc dù tương quan sai số cho thấy đây vẫn là một mô hình tốt, tuy nhiên quy luật không phù hợp. Hàm giả thiết sẽ được thay đổi để bao gồm tất cả các tổ hợp của $\bar{W}$ và $\bar{h}$, hàm có dạng như sau:

$\overline{R_{B}}$

$$
\begin{aligned}
=\theta_{0}+\theta_{1} \cdot \frac{h}{r}+\theta_{2} \cdot \frac{W}{r}+\theta_{3} \cdot\left(\frac{h}{r}\right)^{2}+\theta_{4} \cdot \frac{h}{r} \cdot \frac{W}{r} \\
+\theta_{5} \cdot\left(\frac{W}{r}\right)^{2}+\theta_{6} \cdot\left(\frac{h}{r}\right)^{3}+\theta_{7} \cdot\left(\frac{h}{r}\right)^{2} \cdot \frac{W}{r} \\
+\theta_{8} \cdot \frac{h}{r} \cdot\left(\frac{W}{r}\right)^{2}+\theta_{9} \cdot\left(\frac{W}{r}\right)^{3} \\
+\sum_{i=1}^{d} \sum_{j=0}^{i}\left\{\left(\frac{h}{r}\right)^{i-j} \cdot\left(\frac{W}{r}\right)^{j} \cdot \theta_{T}\right\}
\end{aligned}
$$

Với $T=\left[\left(\sum_{2}^{d} d\right)+1\right] \div\left[\left(\sum_{2}^{d} d\right)+1+d\right]$

Với các tham số như công thức (9), (14) viết dưới dạng ma trận:

Trong đó:

$$
\left\{\dot{\overline{R_{B}}}\right\}=[X] .\{\theta\}
$$

$$
\begin{gathered}
{[X]=\left[\{1\}\left\{\frac{h}{r}\right\}\left\{\frac{W}{r}\right\} \cdots\left\{\frac{h}{r}\right\}^{d} \cdots\left\{\frac{h}{r}\right\}^{i-j} \times\right.} \\
\left.\left\{\frac{W}{r}\right\}^{j} \cdots\left\{\frac{W}{r}\right\}^{d}\right]
\end{gathered}
$$

$[X]$ là ma trận $m \times n$ gồm các thành phần là các véc tơ cột với $\mathrm{i}=1 \div \mathrm{d}, \mathrm{j}=0 \div \mathrm{i} ; \mathrm{n}$ là số đặc trưng của mô hình:

$$
n=\left\{\begin{array}{r}
n_{1=3}, d=1 \\
n_{d}=n_{d-1}+d+a, d \geq 2
\end{array}\right.
$$

Các tham số khác như đã được giải thích trong công thức (10). Cụ thể như công thức (18). Các giá trị (1), (2), ..., (i),... $(m)$ là thứ tự của mẫu trong bộ số $(i=1 \div \mathrm{m})$; số bậc mô hình $j=1 \div d$.

$\left\{\begin{array}{c}\bar{R}_{B}^{(1)} \\ \bar{R}_{B}^{(2)} \\ \vdots \\ \bar{R}_{B}^{(i)} \\ \vdots \\ \bar{R}_{B}^{(m)}\end{array}\right\}_{m \times 1}=$

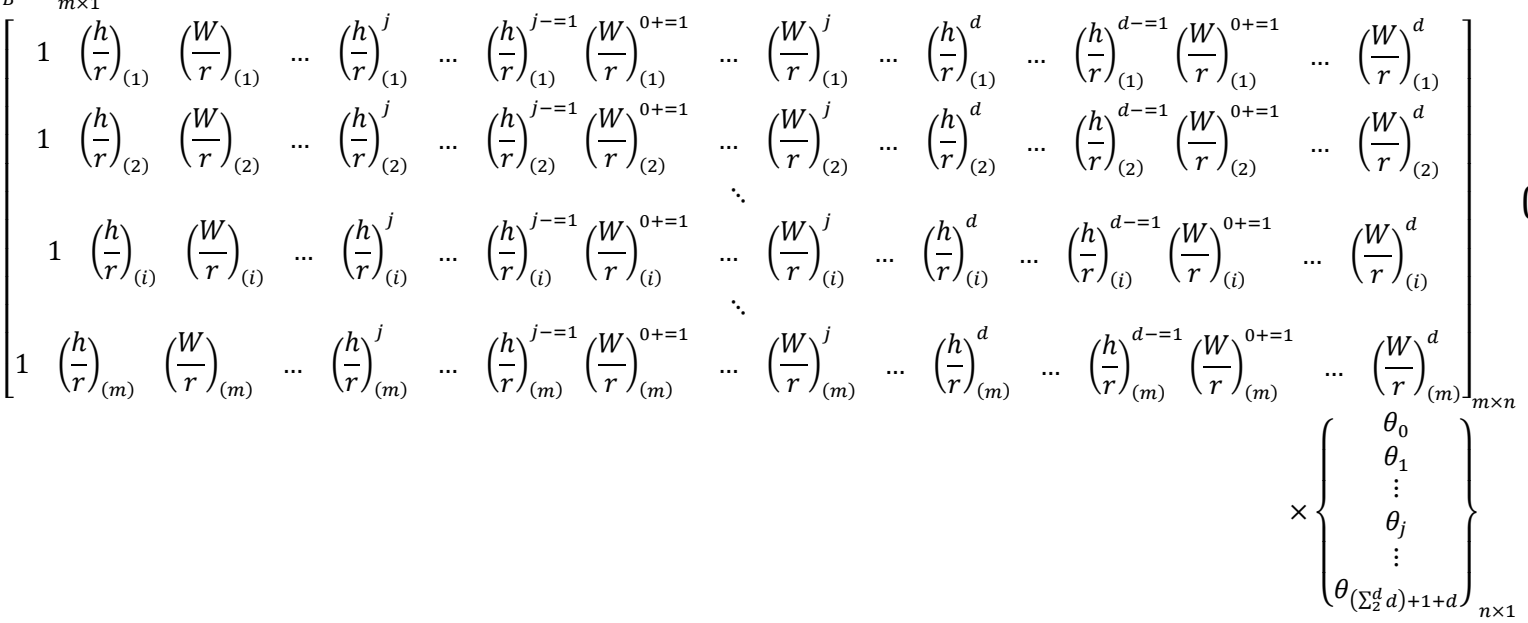

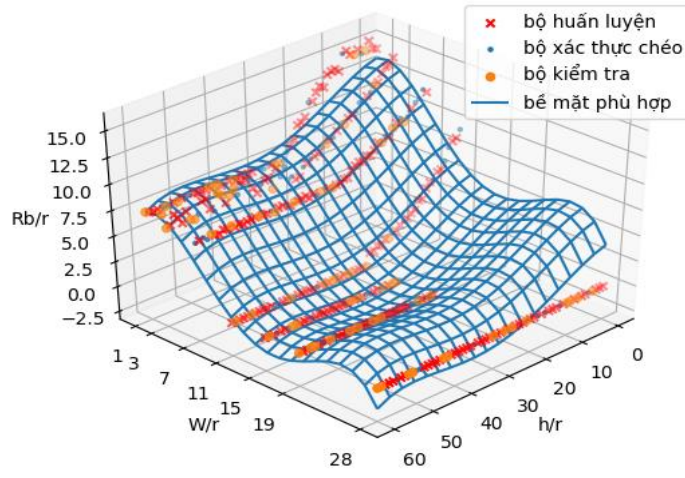

Hình 8. Biểu diễn mô hình $\overline{R_{B}}$ bằng một bề mặt phù hợp ưng với dũ liệu định hướng. 
Sử dụng mô hình có bậc $d=4$, biến tổ hợp cho bộ số định hướng, kết quả trên Hình 9 cho thấy $\lambda=$ 0,01 là phù hợp. Từ (13), kết quả có $R^{2}=0,929$. Bề mặt biểu thị quy luật $\overline{\mathrm{R}}_{\mathrm{B}}$ như Hình 10 và dạng hàm giả thiết được thể hiện ở Bảng 1.

Công thức thực nghiệm quy luật bán kính miệng phễu nổ văng rút ra từ Bảng 1 như sau:

$$
\begin{aligned}
& \frac{R_{B}}{r}=4.28+\frac{-5.42\left(\frac{h}{r}-32\right)}{17}+\frac{-5.38\left(\frac{W}{r}-11.8\right)}{9.16}+ \\
& \cdots+\frac{-21.48\left(\left(\frac{W}{r}\right)^{4}-1.26 \times 10^{5}\right)}{2.26 \times 10^{5}}
\end{aligned}
$$

Tương đương với:

$$
\begin{aligned}
& \frac{R_{B}}{r}=4.28+ \\
& \sum_{j=1}^{14} \frac{\theta_{j}\left\{\left[\left(\frac{h}{r}\right)^{d-=1} \cdot\left(\frac{W}{r}\right)^{d+=1}\right]_{\mid d=1 \div 4}-\mu_{j}\right\}}{\sigma_{j}}
\end{aligned}
$$

Trong đó: $j=1 \div 14$ tương úng trong Bảng $1 ; \theta_{j}$ các tham số ước lượng từ Bảng $1 ; \mu_{j}$ - kỳ vọng hay giá trị trung bình của đặc trung $x_{j}$ trong bộ huấn luyện:

$$
\mu_{j}=\frac{1}{m} \sum_{i=1}^{m} X_{j}^{(i)}
$$
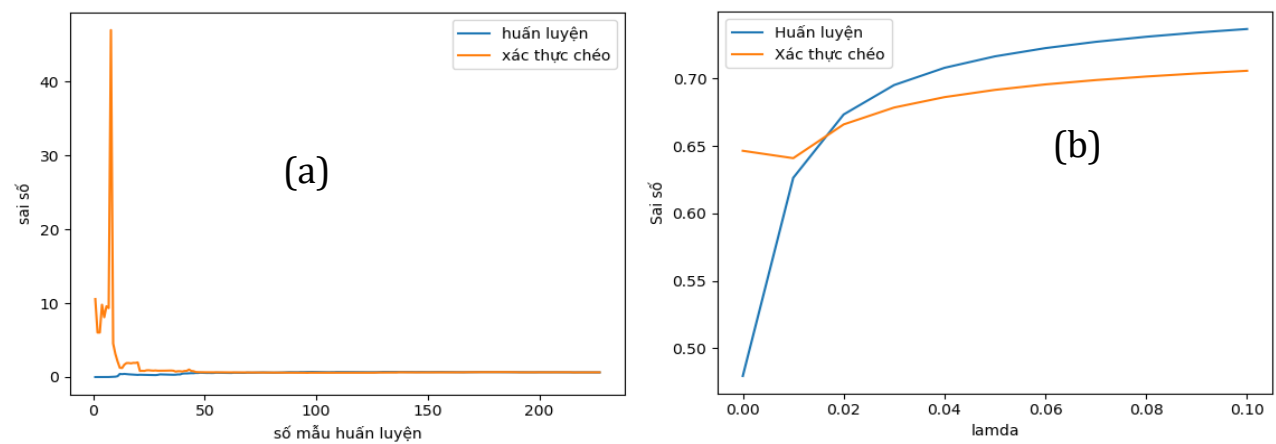

Hình 9. Tưong quan sai số giữa bộ huấn luyện và bộ xác thực chéo với mô hình tổ hợp bậc 4 theo số mẫu huấn luyện (a) và theo $\lambda(b)$ theo dũ liệu định hướng khi lập mô hình $\overline{R_{B}}$.

Bảng 1. Dạng hàm giả thiết được thể hiện trong nghiên cúu này.

\begin{tabular}{|c|c|c|c|c|c|c|c|c|}
\hline \multirow{2}{*}{$\theta_{j}$} & $\theta_{0}$ & $\theta_{1}$ & $\theta_{2}$ & $\theta_{3}$ & $\theta_{4}$ & $\theta_{5}$ & $\theta_{6}$ & $\theta_{7}$ \\
\cline { 2 - 8 } & 4.28 & -5.42 & -5.38 & -2.64 & 2.31 & -16.01 & 12.91 & 3.12 \\
\hline$x_{j}$ & 1 & $\bar{h}$ & $\bar{W}$ & $\bar{h}^{2}$ & $\bar{h} . \bar{W}$ & $\bar{W}^{2}$ & $\bar{h}^{3}$ & $\bar{h}^{2} \cdot \bar{W}$ \\
\hline$\mu_{j}$ & - & $3.20 E+01$ & $1.18 E+01$ & $1.32 E+03$ & $3.93 E+02$ & $2.22 E+02$ & $5.97 E+04$ & $1.62 E+04$ \\
\hline$\sigma_{j}$ & - & $1.70 E+01$ & $9.16 E+00$ & $1.07 E+03$ & $3.82 E+02$ & $2.78 E+02$ & $6.17 E+04$ & $1.94 E+04$ \\
\hline \multirow{2}{*}{$\theta_{j}$} & $\theta_{8}$ & $\theta_{9}$ & $\theta_{10}$ & $\theta_{11}$ & $\theta_{12}$ & $\theta_{13}$ & $\theta_{14}$ & - \\
\cline { 2 - 8 } & -6.15 & 37.54 & -7.40 & -0.34 & -4.37 & 8.23 & -21.48 & - \\
\hline \multirow{2}{*}{$x_{j}$} & $\bar{h} \cdot \bar{W}^{2}$ & $\bar{W}^{3}$ & $\bar{h}^{4}$ & $\bar{h}^{3} \cdot \bar{W}$ & $\bar{h}^{2} \cdot \bar{W}^{2}$ & $\bar{h} \cdot \bar{W}^{3}$ & $\bar{W}^{4}$ & - \\
\hline$\mu_{j}$ & $7.31 E+03$ & $5.05 E+03$ & $2.87 E+06$ & $7.35 E+05$ & $3.00 E+05$ & $1.62 E+05$ & $1.26 E+05$ & - \\
\hline$\sigma_{j}$ & $1.02 E+04$ & $7.97 E+03$ & $3.51 E+06$ & $1.03 E+06$ & $4.90 E+05$ & $2.84 E+05$ & $2.26 E+05$ & - \\
\hline
\end{tabular}

$\sigma_{j}$ - phương sai hay độ lệch chuẩn của đặc trưng $\mathrm{x}_{\mathrm{j}}$ trong bộ huấn luyện:

$$
\sigma_{j}=\sqrt{\frac{1}{m-1} \sum_{i=1}^{m}\left(X_{j}^{(i)}-\mu_{j}\right)^{2}}
$$

$x_{j}$ gồm 14 đặc trưng theo thứ tự từ trái qua phải theo Bảng $1\left(\bar{h}, \bar{W} \ldots . \bar{W}^{4}\right) ; d$ - số mũ cho các đặc trưng $\mathrm{x}_{\mathrm{j}}$, khi $d=1 \div 4$, lần lượt các số hạng trong dấu theo Bảng $1 ; m$ - tổng số mẫu huấn luyện trong bộ huấn luyện; ( $i$ - thành phần thứ $i$ trong bộ mẫu huấn luyện.

Biểu diễn bề mặt phù hợp và tương quan sai số cho thấy đây là một mô hình tốt với độ chính xác cao, phản ánh đúng quy luật nổ.

Từ kết quả thấy rằng hàm giả thiết xây dựng được từ dữ liệu thí nghiệm để phản ánh sự phụ thuộc của bán kính miệng phễu nổ $\overline{R_{B}}$ vào các biến $\bar{W}$ và $\bar{h}$ có độ chính xác cao, từ hình minh họa một bề mặt được lập ra từ mô hình, hoàn toàn có thể sử dụng để dự đoán bán kính miệng phễu nổ $\overline{R_{B}}$ từ mọi giá trị khác nhau của $\bar{W}$ và $\bar{h}$ trong khoảng phạm vi dữ liệu thí nghiệm. Tuy nhiên, mô hình lập ra được học từ những dữ liệu nên dự đoán nội suy sẽ tốt hơn ngoại suy, đồng thời trong thí nghiệm cần thực hiện tìm kiếm biên bão hòa để định hướng mô hình dự đoán đúng quy luật. [ ] trong (18) sẽ bao hàm toàn bộ 14 đặc trưng $x_{j}$ 


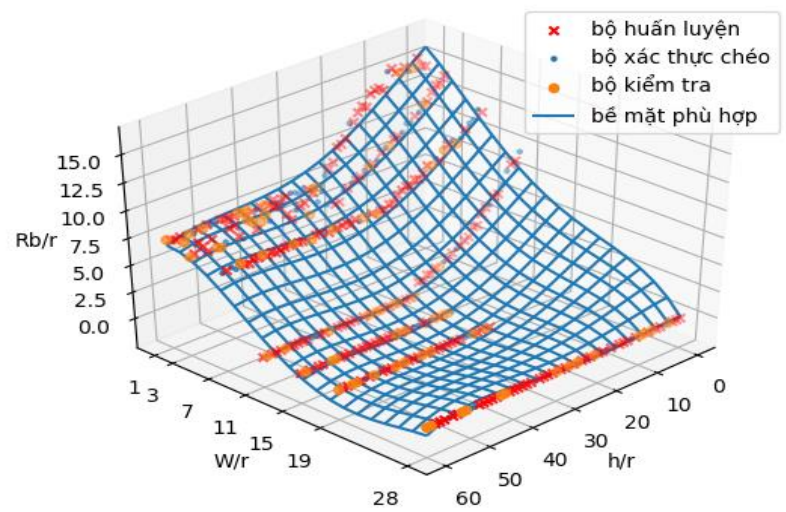

Hình 10. Biễu diễn qui luật phụ thuộc của bán kính phễu nổ văng tương đối $\overline{R_{B}}$ vào chiều sâu đặt thuốc nổ tương đối $\bar{W}$ và chiều sâu nước tương đối $\bar{h}$ biểu diễn ở công thức (18).

\section{Nhận xét, đánh giá}

Từ các đồ thị thể hiện trực quan quan hệ của kích thước phễu nổ văng sau nổ với các đại lượng chiều sâu tương đối, thấy rằng kết quả có độ chính xác cao có thể sử dụng để dự đoán. Tuy nhiên, để đạt được độ chính xác trên thì độ phức tạp trong những hàm giả thiết là rất lớn, kết quả là tạo ra một hàm có rất nhiều tham số.

Phương pháp hồi quy với dạng hàm giả thiết được định trước chưa hiệu quả khi thể hiện những quy luật có độ phức tạp lớn, có tính phi tuyến cao. Với những quy luật phức tạp như vậy, có một phương pháp rất hiệu quả là lập mô hình sử dụng mạng nơ ron nhân tạo với nhiều lớp ẩn, các hàm kích hoạt được áp dụng trong mỗi lớp ẩn có thể giải quyết được những quy luật phi tuyến vô cùng phức tạp.

Phân tích đặc tính phụ thuộc của bán kính phễu nổ văng vào chiều sâu nước và chiều sâu chôn lượng nổ trong đất sét, được thể hiện trong công thức (18) và Hình 10 cho phép rút ra các nhận xét sau:

- Ở vùng giá trị của cặp thông số chiều sâu đặt thuốc và chiều sâu nước nhỏ (gần đúng trong vùng $W / r<11$ và $h / r<(26 \div 30))$, khi tăng một hoặc cả hai thông số thì bán kính phễu nổ văng giảm và ngược lại. Ngoài vùng này nếu tiếp tục tăng một hoặc cả hai thông số chiều sâu đặt thuốc và chiều sâu nước thì bán kính phễu nổ văng đạt trạng thái bão hòa. Các trị số bão hòa gần như bằng nhau ở mỗi mức chiều sâu chôn lượng nổ trong đất sét;

- Ở vùng giá trị của cặp thông số chiều sâu đặt thuốc và chiều sâu nước lớn (gần đúng trong vùng $W / r>11$ và $h / r>(0 \div 30))$, khi tăng một hoặc cả hai thông số thì bán kính phễu nổ văng bằng không. Hiện tượng nổ ngầm bắt đầu xuất hiện.

\section{Kết luận}

Bán kính phễu nổ văng phụ thuộc vào ba thông số chiều sâu đặt thuốc trong môi trường đất sét, chiều sâu nước và bán kính lượng nổ. Qui luật chung bán kính phễu nổ văng dưới nước phụ thuộc đồng biến với bán kính lượng nổ và phụ thuộc nghịch biến với chiều sâu chôn lượng nổ trong môi trường sét và chiều sâu nước, khi nổ trong vùng có hình thành phễu nổ văng. Khi nổ trong vùng bão hòa hoặc nổ ngầm, tương ứng với nổ ở độ sâu lớn thì bán kính phễu nổ văng là hằng số không phụ thuộc vào chiều sâu chôn lượng nổ hoặc chiều sâu nước.

Mô hình hồi qui được xây dựng từ những thuật toán máy học đã học từ những dữ liệu để tạo nên một mô hình giả thiết miêu tả tương đối chính xác quy luật phụ thuộc của bán kính phễu nổ văng vào chiều sâu đặt thuốc trong đất sét, chiều sâu nước và bán kính lượng nổ. Tuy nhiên để nhận được qui luật hồi qui phù hợp với qui luật tác dụng nổ, thì ngoài việc cần một bộ số liệu đầy đủ, chính xác, còn cần có sự bổ sung định hướng bộ số liệu thứ cấp là rất cần thiết để việc tìm ra quy luật được khách quan và đúng với thực tế. Qui luật tìm ra có ưu điểm là một hàm liên tục đa biến so với phương pháp giải tích truyền thống.

\section{Đóng góp của các tác giả}

Tác giả Đàm Trọng Thắng hình thành ý tưởng, nội dung và hoàn thành bản thảo cuối của bàiáo; tác giả Vũ Tùng Lâm thu thập số liệu, đọc bản thảo trung gian. 


\section{Tài liệu tham khảo}

Belin, V. A., Dam Trong Thang, (2006). Empirical study of splashing explosives charges which put on the bottom of water, for digging canals and ditches under water, General scientific journal No 11, Moscow, May 2006. (Translated from Russian).

Dam Trong Thang, (2006). Studying on reasonable parameters of splashing explosives charges for building construction under water in Vietnam. Doctoral thesis - MGGU 2006. (Translated from Russian).

Dam Trong Thang, Belin V. A., Nguyen Thanh Dong, (2019). Empirical study of formation process of splashed funnel and the smoldered blasting effect when blasting the concentrated explosives charges under water. UDK622.235 -Science and technology journal of "Blasting Works", No 122/79, IPKON RAN publisher, Moscow 2019. (Translated from Russian).

Galkin, V. V., Gilmanov, R. A., Drogoveyco I. Z., (1987). Blasting works under water. Underground publisher, Moscow. (Translated from Russian).

Baum, F. A., Staniukovic, K. P., Sekhter, B. I., (1975). Physics of explosion. Puplisher of National math and physics, Moscow. (Translated from Russian).

Vlaxov, O. E., (1975). Theoretical basis of explosion effects. VIA publisher, Moscow. (Translated from Russian).

Pokrovskii, G. I., Phedorov, I. S., (1957). The impact of shock and deformation in the environment. Publisher of National construction, Moscow, 1957. (Translated from Russian).

Sedov, L. I., (1945). Similarity method and dimensionality in mechanics. GITL publisher, Moscow. (Translated from Russian).

Romaxov, A. N., (1980). Argumentation of the effects of large underground explosions. Underground publisher, Moscow, (Translated from Russian).

Criukov, G. M., (1985). Physics and the demolition of explosion. MGI publisher, Moscow, 1985. (Translated from Russian).

Kutuzov, B. N., (1990). Empirical works for subject "Demolishing the ground with explosions". MGI publisher, Moscow. (Translated from Russian).

Novikov, V. D., Luchko, I. A., Iurich, I. I., Gliaskii, G. I. A., (1984). Argumentating formation process of blasting funnel under water. Kiev publisher. (Translated from Russian.

Gorodinov, L. V., (1993). Studying on mechanical effects of external explosives charges in submerged rock and soil. Brief doctoral thesis, Novosibirisk. (Translated from Russian).

Kutuzov, B. N., Ruptsov, V. K., (1970). Physics of demolishing the ground with explosions. Chapter 1, MGI publisher, Moscow. (Translated from Russian).

Tavrivov, V. M., (1949). Digging into the seabed with explosions. Publisher of Minischerva Rechnovo Pnota CCCP, Moscow. (Translated from Russian).

Josef Henrych, (1979). The dynamic of explosion and its use. Academia Prague, 1979.

Vu Huu Tiep, (2018). Basic machine learning. Publisher of science and technology. (Translated form Vietnamese).

Andrew Ng, (2011). Machine learning online course at www.coursera.org, 2011. 